\title{
Effects of Rotation on Helium Accreting White Dwarf
}

\author{
S.-C. Yoon and N. Langer \\ Astronomical Institute, Utrecht University, Princetonplein 5, 3584 CC, \\ Utrecht, The Netherlands
}

Classical studies of accreting white dwarfs have assumed spherical symmetry. However, it is believed that in close binary systems the transfered matter carries angular momentum to spin up the accreting star. Here, we present preliminary results of $\mathrm{CO}$ white dwarf models which accrete helium rich matter with effects of rotation considered, in the context of the Sub-Chandrasekhar mass scenario for Type Ia supernovae.

The numerical models have been computed with a hydrodynamic stellar evolution code in which we include the effect of the centrifugal force on the stellar structure, and rotationally induced transport of angular momentum and chemical species (Heger et al. 2000). Matter is accreted at a constant rate of $2 \times 10^{-8} \mathrm{M}_{\odot} \mathrm{yr}^{-1}$, containing a helium and ${ }^{14} \mathrm{~N}$ mass fraction of $Y=0.981$ and $X_{\mathrm{N}}=0.012$. The specific angular momentum of the accreted matter is assumed to have the Keplerian value at the white dwarf equator as long as the white dwarf surface does not rotate over-critically (see Yoon et al. 2003). The rotating white dwarf models are spun up by the angular momentum gain from the accreted matter. The resultant differential rotation may cause frictional energy dissipation, which we estimate with $\epsilon_{\text {diss }}=\frac{1}{2} \nu\left(r \frac{d \omega}{d r}\right)^{2}$ (e.g. Kippenhahn $\&$ Thomas 1978, Mochkovitch \& Livio 1989), where $\nu$ is the turbulent viscosity due to rotationally induced instabilities.

Table 1. $M_{\text {init }}$ : initial mass in $\mathrm{M}_{\odot}, \Delta M_{\mathrm{He}}$ : accreted helium mass at helium ignition in $\mathrm{M}_{\odot}, \psi$ : degree of degeneracy at the helium ignition point, $\rho_{\text {ig: }}$ : density at the helium ignition point in $\mathrm{g} / \mathrm{cm}^{3}$.

\begin{tabular}{c|ccc|ccc|ccc}
\hline & \multicolumn{3}{|c}{ No Rotation } & \multicolumn{3}{c}{ Rotation with $\epsilon_{\text {diss }}$} & \multicolumn{3}{c}{ Rotation without $\epsilon_{\text {diss }}$} \\
\hline \hline$M_{\text {init }}$ & $\Delta M_{\mathrm{He}}$ & $\psi$ & $\rho_{\text {ig }}$ & $\Delta M_{\mathrm{He}}$ & $\psi$ & $\rho_{\text {ig }}$ & $\Delta M_{\mathrm{He}}$ & $\psi$ & $\rho_{\text {ig }}$ \\
\hline 0.6 & 0.24 & 24 & $1.7 \times 10^{6}$ & 0.20 & 7 & $3 \times 10^{5}$ & - & - & - \\
0.8 & 0.17 & 23 & $1.6 \times 10^{6}$ & 0.02 & 5 & $2 \times 10^{5}$ & 0.3 & 17 & $10^{6}$ \\
\hline
\end{tabular}

Our results are summarized in Table 1. In the non-rotating models, the helium ignition is triggered by the ${ }^{14} \mathrm{~N}\left(\mathrm{e}^{-}, \nu\right)^{14} \mathrm{C}(\alpha, \gamma){ }^{18} \mathrm{O}$ reaction when the mass of the accreted helium reaches $\sim 0.2 \mathrm{M}_{\odot}$. At this point, the helium flash occurs in highly degenerate conditions likely leading to a helium detonation and a supernova explosion is expected (Woosley \& Weaver 1994). 


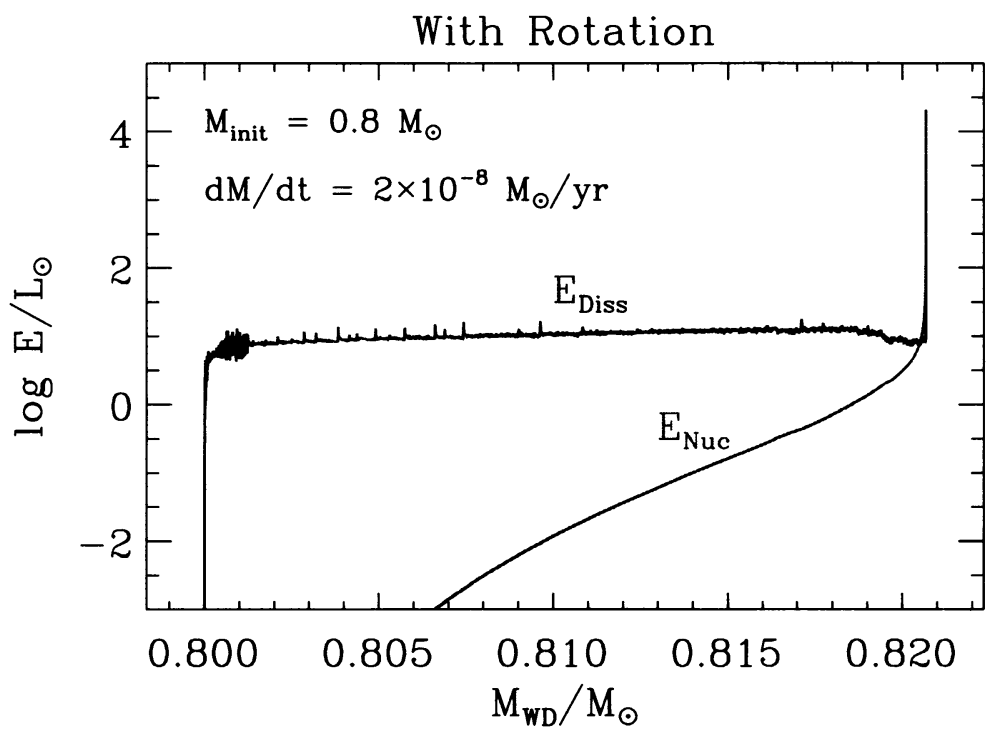

Figure 1. Evolution of luminosity due to rotational energy dissipation (indicated by $\mathrm{E}_{\text {diss }}=\int \epsilon_{\text {diss }} d M_{r}$ ) and nuclear energy generation (indicated by $\left.\mathrm{E}_{\text {nuc }}=\int \epsilon_{\text {nuc }} d M_{r}\right)$ in the rotating white dwarf model with $M_{\text {init }}=0.8 \mathrm{M}_{\odot}$ and $\dot{M}=2 \times 10^{-8} \mathrm{M}_{\odot} \mathrm{yr}^{-1}$, as function of the total mass of white dwarf which serves as a measure of time. The helium flash occurs when the white dwarf has accreted $0.02 \mathrm{M}_{\odot}$ of helium.

In the rotating models where we include frictional energy dissipation due to the differential rotation, the white dwarf interior is heated up efficiently (Fig. 1). As a result, the helium flash occurs earlier and in much less degenerate conditions than in the corresponding non-rotating cases or in the rotating model without frictional energy dissipation (Table 1). As the helium ignition density and degeneracy are strongly reduced in the rotationg models if frictional energy dissipation is considered, a helium detonation may be avoided. Since a helium detonation seems required to obtain a supernova explosion, our models question the sub-Chandrasekhar mass scenario for Type Ia supernovae as a whole.

\section{References}

Heger A., Langer N., Woosley S.E. 2000, ApJ 528, 368

Kippenhahn R., Thomas H.-C. 1978, A\&A 63, 265

Mochkovitch R., Livio M. 1989, A\&A 209, 111

Woosley S.E., Weaver T.A. 1994, ApJ 423, 371

Yoon S.-C., Langer N., Scheithauer S. 2003, A\&A in preparation 